Case Report

\title{
AN UNUSUAL PRESENTATION OF LANGERHANS CELL HISTIOCYTOSIS
}

\author{
Jayaprakash Shetty K. ${ }^{1}$, Ajith Kumar ${ }^{2}$, Shantharam Shetty ${ }^{3}$, Kishan Prasad H.L. , Chandrika Rao ${ }^{1}$ \\ ${ }^{1}$ K.S. Hegde M edical Academy of Nitte University, ${ }^{2}$ Senior Consultant Orthopaedician, Tejaswini hospital, \\ ${ }^{3}$ Pro-Chancellor of Nitte University, \\ Institution : KS Hegde Medical Academy of Nitte University, M angalore, Karnataka, India.
}

\section{Correspondence: \\ Jayaprakash Shetty K.,}

Professor and HOD, Department of Pathology, K.S. Hegde Medical Academy of Nitte University, Deralakatte, Mangalore, Karnataka, India. E-mail : drjpshetty9@ rediffmail.com

\begin{abstract}
:
Langerhans cell histiocytosis most commonly occurring in children, demonstrates a broad spectrum of clinical and radiologic features that may mimic those of infection as well as benign and malignant tumors. Osseous involvement is the most common manifestation. Recognition of the skeletal alterations is important so that the disease is considered as differential diagnosis. This disease is of unknown aetiology, non-specific clinical and imaging findings with diagnosis possible only on histopathological examination, will always pose a diagnostic challenge to the orthopaedic surgeon. Here by we are reporting two cases of eosinophilic granuloma that has presented with lytic lesion in the tibia and scapula. The clinico-radiological suspicion was either infection or tumour of the affected bones. Hence, core biopsy was done confirmed as eosinophilic granuloma on histopathology and immunohistochemistry.
\end{abstract}

Keywords: eosinophilic granuloma; tumour; osteomyelitis; CD 1a.

\section{Introduction :}

Langerhans cell histiocytosis $(\mathrm{LCH})$, previously called as histiocytosis $X$, refers to a spectrum of disease characterized by idiopathic proliferation of histiocytes producing focal or systemic manifestations. The recent studies suggested abnormal immune regulation as an important factor. Among these, eosinophilic granuloma (EG) commonly presents with skeletal manifestation. Neither the clinical nor the radiographic presentation of EG is specific, and thus poses diagnostic dilemma to the orthopaedic surgeon. ${ }^{1,2,3,4}$

Osseous involvement is typically in the flat bones, with lesions of the skull, pelvis, and ribs accounting for more than half of all lesions. About $30 \%$ of lesions are in long bones and it rarely \begin{tabular}{l} 
Access this article online \\
\hline Quick Response Code
\end{tabular} manifests as tibial lytic lesion. The commonest differential diagnosis made in an adolescent patient with a relatively short history of severe bone pain and bony tenderness in the metaphyseo-diaphyseal region of a long bone is either infection or tumour. Newer investigation modalities like computed tomography and magnetic resonance imaging helps in evaluating the extent of the lesion but the diagnosis could only be confirmed by histopathology. ${ }^{2,34}$ Here by we are reporting two cases of eosinophilic granuloma which has presented with lytic lesion in the tibia and scapula.

\section{Case Reports:}

Case 1; A 3-year-old child came with right leg pain since one week. There was no history of trauma. $X$ ray showed, lytic lesion in the proximal tibia[Fig $1 \mathrm{~A}]$. Clinical diagnosis of osteomyelitis or ewings sarcoma was made. Hence, core needle biopsy was done showed aggregates of eosinophils, lymphocytes and Langerhans cell histiocytes with grooved nuclei [Fig 2A and 2B] suggestive of eosinophilic granuloma. Immunohistochemistry showed strong immunoreactivity for CD 1a, CD 68 and S100 [Fig 3A, 3B, 3C] hence confirming the diagnosis.

Case 2; A 3-year-old old child presented with inability to move the right upper limb, found to have diffuse lytic lesion 


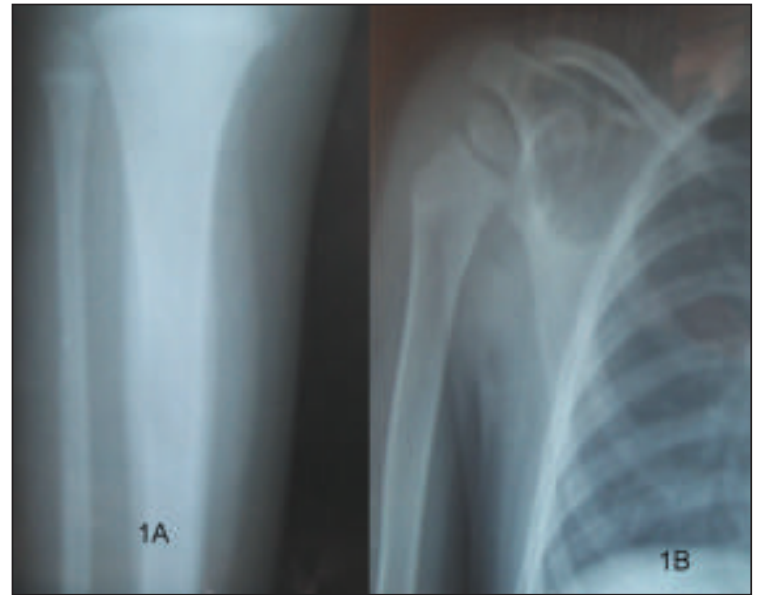

Fig 1 : 1A showing lytic lesion in the diaphysis of tibia Fig 1B showing diffuse lytic lesion in the proximal scapula

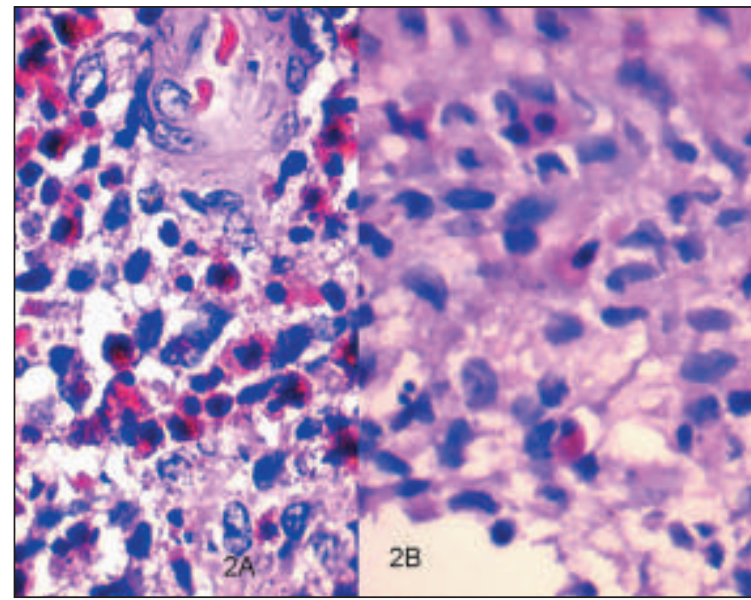

Fig2: 2A showing mixed inflammatory cells cheifly eosinophils Fig 2B showing aggregates of langerhan cells $\mathrm{HE}$.

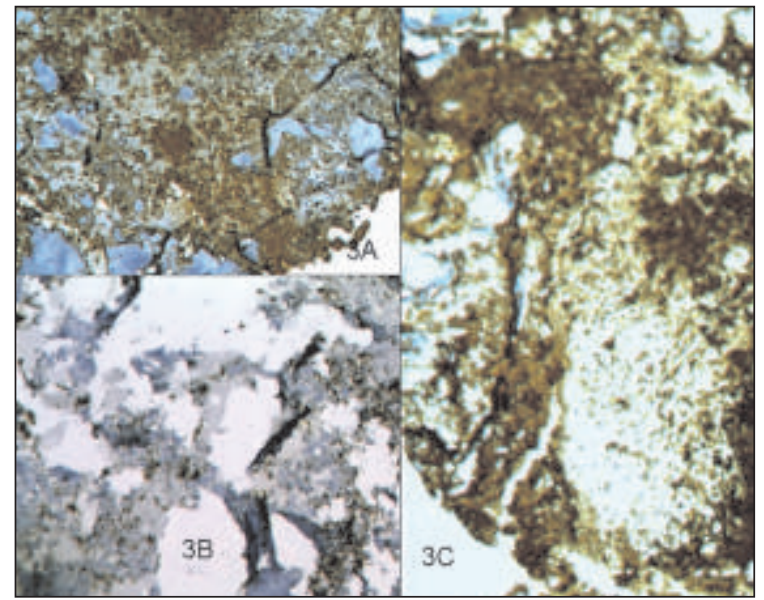

Fig 3 : 3 Immunohistochemistry showing immunoreactivity for $C D$ 1a 3A CD 683 B and S1003C. in the scapula[Fig 1B]. The core biopsy confirmed as eosinophilic granuloma on histopathology and immunohistochemistry.

\section{Discussion :}

Solitary EG of bone is the commonest manifestation of histiocytosis (60 to $80 \%$ cases). The hallmark of histiocytosis $X$ is the Langerhans cell, a histiocyte with characteristic racquet shaped Birbeck granules visible on electron microscopy. The pathogenesis of EG is not completely understood. Infections, immune and neoplastic causes have been postulated. ${ }^{1,3}$ Up to $80 \%$ of histiocytosis Xlesions in children are of solitary EG type and up to $90 \%$ occur in children. ${ }^{1,4,5}$ The three classic syndromes may have considerable clinical overlap: Eosinophilic granuloma, in which the disease is limited to bone in patients of 5-15 years old, Hand-Schuller-Christian disease, characterized by multifocal bone lesions and extraskeletal involvement of the reticuloendothelial system (RES) usually seen in children 1-5 years old; and Letterer-Siwe disease, in which there is disseminated involvement of the RES with a fulminant clinical course in children less than 2 years old. ${ }^{1,3,4}$

In histopathology of $\mathrm{LCH}$, the unique histiocytes form granulomas in conjunction with lymphocytes, polymorphonuclear cells and eosinophils. During the early phase of $\mathrm{LCH}$, lesions are cellular and are marked by aggregates or sheets of langerhans. Eosinophils are often identified. Older LCH lesions, which may be mistaken for chronic osteomyelitis,

myelofibrosis, or "non-specific benign/fibrous lesions", are marked by a paucity of langerhans and a fibrous background, with or without eosinophils. ${ }^{1,2,3,4}$ Immunohistochemistry shows strong immunoreactive for CD 1a, CD 68 and $\mathrm{S} 100$ similar to our case.

The treatment of EG is equally controversial with different therapeutic approaches claiming effectiveness. Solitary EG of bone has been shown to undergo spontaneous remission. ${ }^{1}$ Symptomatic surgically accessible solitary EG are managed by biopsy, curettage and bone grafting if 
needed. ${ }^{2-4}$ A single curettage usually results in healing. ${ }^{1}$ Local injection of corticosteroids was described by Scaglietti et al, with immediate pain relief and healing response within 2 months after injection. ${ }^{3-5}$ Irradiation is rarely used because of reports of late latent neoplasms. ${ }^{1-4}$ The use of chemotherapy and oral corticosteroids alone or combined is indicated in systemic disease but rarely in a solitary lesion due to unpredictable results obtained and the toxic and oncogenic risk. ${ }^{3}$ It has been stated that patients with unifocal EG, who subsequently develop additional lesions, usually do so within 6 months after

\section{References:}

1. Moon TY, Lee J, Lee IS, Choi KU, Chae JM, Kim J et al, MRI and Histopathologic Classification of Langerhans Cell Histiocytosis. Current M edical Imaging Reviews 2009;5:14-18.

2. Chadha M, Agarwal A, Agarwal N, Singh MK. Solitary eosinophilic granuloma of the radius. An unusual differential diagnosis. Acta Orthop Belg 2007;73:413-7.

3. Plasschaert F, Craig C, Bell R, Cole WG, Wunder JS, Alman BA. Eosinophilic granuloma. A different behaviour in children than in adults. J Bone Joint Surg 2002;84:870-2.

4. M cgravan M H, Spady HA. Eosinophilic granuloma of Bone. A study of twenty-eight cases.J Bone Joint Surg 1960;42:979-82.

5. Stull MA, Kransdorf MJ, Devaney KO. Langerhans Cell Histiocytosis of Bone. Radiographics 1992;12:801-23. appearance of the initial lesion. ${ }^{2}$ Other favourable prognostic factors are age greater than 2 years, absence of pulmonary, hepatic, haemopoietic lesions or multiple bony involvement. $^{2}$

\section{Conclusion:}

The LCH with its confusing nomenclature, unknown aetiology, non-specific clinical and imaging findings with diagnosis possible only on histopathological examination, will always pose a diagnostic challenge to the orthopaedic surgeon. We conclude with a single message. Suspect it, lest you will missit. 